\title{
Study and application of the fully insulated bench transformer substation of standard building blocks
}

\author{
Wang Gang \\ Liaoning Electric Power Co., Ltd. \\ w.g100@163.com
}

Keywords: power distribution; full insulation; bench substation; standardization

\begin{abstract}
In the distribution network, pole-type transformer is not only the most common device related to power supply safety and environmental beautification, but also in a large amount of number and needs a large number of maintenance. It is difficult to achieve the standardization. In view of the existing problems of the original power transformer substation, such as the poor ability to resist natural disasters, unreliable operation, low intelligence level, non insulation, easy corrosion, technological backwardness, and incoordinated with the environment, the fully insulated transformer substation of standard building blocks is developed by adopting the idea and method of industrial design. The new type bench substation, which is fully insulated and maintenance-free with advanced and reliable materials, is safe, aesthetic, light, simple, corrosion resistant, and environmental protected. It further protects the safety of personnel and equipment, and it is convenient to operate and manage. This paper describes the study contents, technical route, function and application effect of the new type transformer substation respectively.
\end{abstract}

\section{Introduction}

With the rapid development of urban construction and people's new requirements for the modernization of new city, it is a necessary trend to plan and reform the new type power distribution device to improve and optimize the structure of the distribution network and provide quality and reliable power for the development of urban construction and people's production and life. The original pole-type transformer, with high failure rate and short service life, has a wide variety of structures without a uniform standard, needs a large amount of maintenance, and is not coordinated with the environment. When China tries to promote energy conservation and emission reduction and revitalize new energy and other emerging industries, according to the general thought of "building smart grid" proposed by China State Grid Corp, the current situation of domestic distribution device, and these problems of the original pole-type transformer, the company, in the purpose of serving the end customers, actively organizes technical innovation, creates a new distribution technology revolution, takes the use of the ideas and methods of industrial design, and takes the lead in launching a new type of bench substation of intelligent building blocks in the country. Under the premise of ensuring safe operation and meeting the requirement of power supply, the new type transformer substation, by setting up a new concept, and actively adopting new technology, new material, new equipment and new technical process, has improved the overall function and the appearance of the original transformer substation completely, and laid a good foundation for improving the standardization and intelligence of the distribution device.

\section{Study content}

The fully insulated bench transformer substation of standard building blocks uses modular combination mode. Its structure is divided into four modules, including: bench structure, ingoing and outgoing line of high and low voltage, transformer, and low voltage load control box.

\section{1) Bench structure}

The bench adopts the "H" mode, the bottom of the bench is 3.5 meters from the ground, and the bottom of the load control box is 2.5 meters from the ground. The size of the bench substation and the auxiliary facilities are reduced, which beautifies the city environment. 
Pole combination:

The mode of low voltage overhead line distribution. The main pole and auxiliary poles use cement poles of equal height; the main pole and auxiliary poles of transformer bench are equipped with a working ladder, to facilitate the construction personnel in operation, installation and maintenance.

The mode of low voltage cable distribution. The main pole and the auxiliary poles use the cement poles at different height, and the main pole is equipped with a working ladder.

The frame of the fully insulated bench transformer substation of standard building blocks selects the new insulation materials ( the enhanced composite glass fiber process), which has the advantages of corrosion resistance, light quality, environmental protection and security. The base of the bench substation is a multifunctional maintenance bench, which can be used as a platform for maintenance, and can be used as the interlayer of high and low voltage cables.

Four-point suspension installation method is adopted for the low voltage load control box.

\section{2) High and low voltage wiring}

The high voltage switch selects the imported fuse protector of high strength porcelain and drop type, and the breaking current is 12.5KA. The drop type fuse protector is installed in the top of the main pole to avoid the scratches and bumps of past vehicles.

The down lead of high voltage selects single core cable, which is fixed along the pole down through the insulating cable tray to the insulation interlayer of the bench by the special fixture. The cable comes through the insulation interlayer of the bench to the high voltage bushing side of the transformer, and is connected to the transformer by the elbow cable plug. High voltage lighting arrester uses zinc oxide arrester, the down lead of the arrester is discharged to the cable groove through the transition busbar. The suspension point of high voltage grounding wire adopts the integrated grounding suspension device of special plastic insulation. The down lead of the lower part of the bench substation adopts JBYJ type insulation grounding poles and is fixed by stainless steel banding. The insulated soft copper wire is used from the transformer to the low voltage outgoing line of the load control box, and the low-voltage insulation line is used from the load control box to the low voltage main line, and they are fixed to the low voltage lines along the pole up through the low-voltage line tray by the special fixture (if low voltage cable outlet is guided down by the load control box, the composite type glass fiber reinforced protecting pipe is used and the protective tube is fixed by the special fixture).

\section{3) Transformer}

The oil immersed fully insulated transformer of energy-saving type is used, which is convenient in construction with aesthetic appearance, the high and low voltage side is set the door type maintenance cover, and ingoing and outgoing lines are closed. The attached low voltage bench area is measured and assessed by combined type current transformer, which is convenient to adjust the mutual inductor when the transformer is replaced.

\section{4) Low voltage load control box}

The shell of the low voltage load control box is made of high quality SMC material and the box, with two side opening doors, is divided into five units, including the main load, the load, measurement, compensation and the frame unit of ingoing and outgoing lines. The switch of the ingoing line uses the load isolation switch, while the switch of the outgoing line uses the moulded case circuit breaker. The reactive compensation of low voltage adopts the automatic switching device with controllable silicon. The ingoing and outgoing lines of the load control box use frame unit jacket of ingoing and outgoing lines. The intelligent low voltage control box, with warning signs, is equipped with GPRS electric power meter and an electric energy meter inside, which has the function of collecting data by GPRS in the field. It has the automatic control interface, which can be used to determine the operating parameters and power information of the transformer substation to provide scientific basis for operation and management. 


\section{Technical route}

The fully insulated bench transformer substation of standard building blocks is with superior functions, novel structure, advanced materials, convenient construction. The main technical routes are as follows:

1) each component are produced in the idea of industrialization, and the main parts of the bench substation use mouled by mold, and are designed and produced by modular, to ensure the consistency and the exchange of the components, and all parts take the use of modular design. The installation site does not need welding, power, and it can be directly assembled, easy to install and maintain.

2) all the materials of bench substation, including the cable tray of high and low voltage, bench, protective tube and holding hoop, adopt new high strength non metal material of FRP, and the shell of the load control box adopts SMC insulation material, which is insulated, corrosion resistant, environment protective, aesthetic, light, durable and easy to install.

3 ) the structure of every component is compact and the overall layout is reasonable, and cable protection of the high and low voltage is fully considered. It, with aesthetic appearance and good insulation, is protected by the fully insulated cable tray to ensure that external line does not leaked, so that it ensures the safety and coordination with the environment.

\section{Technological breakthrough}

1) Advanced materials. The advanced materials truly realize the full insulation. All the bench, the cross bear, the busbar and the load control box take the use of new materials to further protect the safety of personnel and equipment. The application of the fully insulated bench substation is still the first case in China.

2) Aesthetic appearance. With compact structure of every component, and reasonable layout, it uses the indoor wiring method and the high and low voltage leads are laid by the insulated busbar. It is simple, aesthetic, and coordinated to environment.

3) Innovated programme. New style fully insulated substation of building block applies a number of technologies and innovations, such as the new bench, integrated suspension point device of high voltage grounding wire, multifunction maintenance platform, fully insulated transformer, insulation protection grounding pole, outdoor insulation busbar, and cable insulation protection tube.

4) Superior performance. The selection of the submission targets free maintenance, energy saving and emission reduction. It uses advanced technology, modular production, to ensure its superior technical performance.

5) Convenient construction. Each part of the bench uses the form of building blocks, which are simple to install, and the transformer leads are with a new design, which is simple and convenient.

\section{Application Management}

The fully insulated bench transformer substation of standard building blocks which is applied in Liaoning Electric Power Co., Ltd for the first time, has passed the professional acceptance of Liaoning Electric Power Co., Ltd, and the type test of such authority testing departments as Northeast Electric Power Study Institute and Shanghai Glass Steel Study Institute and meets the national industry standards(1)(2).

The fully insulated bench transformer substation of building blocks has been successfully used for 6 years, and has been promoted in Liaoning province and some other provinces and cities in the country. It went through the cold winter and hot summer, especially the "2.25" freezing rain and "7.21" rainstorm in Liaoning Province in 2010; its new design concept has always being praised by the front-line staff. Owing to its fully insulated and sealed structure, its effect of preventing and resisting natural disasters is outstanding, and the failure rate has dropped significantly; it is 
maintenance free, which reduces the personnel's operation risk; it is aesthetic and environmental coordinated. The design scheme has been incorporated into the typical design of the China State Grid Corp and the successful operation of the new substation of building blocks has laid a good foundation for the construction of a strong intelligent power distribution network.

\section{Function and benefit}

The construction of the new style standard fully insulated bench transformer substation of building blocks, with hug professional technical and social economic value, promotes and standardizes the standard of the power distribution device and realizes the standard and intelligent management of the transformer substation.

\section{1) Professional technical value}

The standardization of the power transformer substation has got new breakthrough. The main advantages of the new style fully insulated bench transformer substation of standard building blocks decide the direction of its future distribution. The advantage is that it entirely uses the insulation materials, all the connecting parts are positioned at 4 points, which ensures the overall non-deformation, intensive mechanical strength, high stability, overall appearance, high temperature resistance and corrosion resistance.

Except for the ladder, the fixture parts of the bench transformer substation use the stainless steel bolt for connection, so that the aesthetics and non corrosion are ensured. Low voltage intelligent control box uses SMC insulation material, which is thick, insulated, aging resistant and stable. The switch in the box has function of short circuit and grounding protection. Power collector should have the function of collecting the low voltage data, and keep the automatic control interface, in order to realize the control and protection remote load in the future.

\section{2) Social value}

It is safe, environment protective and coordinated with the environment. The new style fully insulated bench transformer substation of standard building blocks is unique for its safe and reliable structure, aesthetic appearance, and less impact on the environment and personnel, so it is coordinated with urban construction.

\section{3) Economic value}

It is maintenance free with low failure rate. Compared with the traditional bench substation, the new style fully insulated bench transformer substation of standard building blocks has a high reliability and long service life, greatly reducing maintenance cost and power failure loss.

\section{Conclusions}

The new style fully insulated bench transformer substation of standard building blocks has been used since 2009 in Liaoning Electric Power Co., Ltd. During the 6 years, it has received consistent praise from the users, and the current operation of the device is stable. With advanced and reliable materials, the new type transformer substation is really safe, aesthetic, simply installing, and free of maintenance, which further ensures the safety of personnel and equipment and is convenient in operation and management. The whole design is applied in the country for the first time. Under the premise of ensuring safe operation and meeting the requirement of power supply, the new type bench substation has set up a new idea, improved the whole function and appearance of the original station, and laid a good foundation for improving the standardization and intelligence of distribution device.

\section{References}

[1]GB/T16927-1997 High voltage test technique

[2]GB/T1410-2006 Test method of volume resistivity and surface resistivity of solid insulating materials 
[3]Q/TXS0001-2012 Enterprise standards of fully insulated transformer substation of building blocs [4]State Grid Corporation of China. The power supply enterprise safety risk assessment standard[M].Beijing:China Electric Power Press,2009.

[5]SAATY $\mathrm{T}$ L. Fundamentals of the analytic hierarchy process[M].Pittsburgh: RWS Publications,2000.

[6]DAGDEVIREN M,YUKSEIJ. Developing a fuzzy analytic hierarchy process(AHP)model for behavior—based safety management[J].Information Sciences,2008,178(6):1717- 1733. 